\title{
Visnagin ameliorates myocardial ischemia/reperfusion injury through the promotion of autophagy and the inhibition of apoptosis
}

\author{
Hai-rong Fu, ${ }^{1}$ Xiao-shan Li, ${ }^{1}$ Yong-hui Zhang, ${ }^{1}$ Bin-bin Feng, ${ }^{2}$ Lian-hong Pan ${ }^{3}$ \\ ${ }^{1}$ Department of Basic Medicine \\ ${ }^{2}$ Department of Pharmacy \\ ${ }^{3}$ Center for Natural Anti-tumor Medicine Engineering, Three Gorges Medical College, Chongqing, China
}

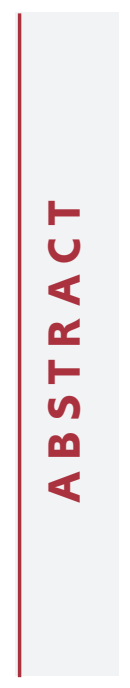

\begin{abstract}
Visnagin is a furanochromone and one of the main compounds of Ammi visnaga L. that had been used to treat nephrolithiasis in Ancient Egypt. Nowadays, visnagin was widely used to treat angina pectoris, urolithiasis and hypertriglyceridemia. The potential mechanisms of visnagin involved in inflammation and cardiovascular disease were also identified. But the protective effect of visnagin on myocardial ischemia/reperfusion injury has not been confirmed. Our aim was, for the first time, to investigate the potential protective effect of visnagin on cardiac function after myocardial ischemia-reperfusion injury in a rat model, and to identify its underlying mechanism involving the inhibition of apoptosis and induction of autophagy. Thirty SD rats were randomly divided into sham group, ischemia/reperfusion group (IR), ischemia/reperfusion with visnagin (IR + visnagin) group. Myocardial ischemia/reperfusion injury model was established. Hemodynamic measurements and echocardiography were used to analyze cardiac function; TUNEL staining and caspase activity, LC3 dots were detected with immunofluorescence staining, LC3 expression was evaluated by western blot analysis; transmission electron microscopy was used to detect autophagosomes. Compared with the sham group and visnagin group, the cardiac dysfunction; LC3II, autophagy flow in the IR + visnagin group increased significantly $(\mathrm{P}<0.01)$, but the activity of caspase- 3 and caspase- 9 and the apoptotic index in the IR + visnagin group decreased significantly $(\mathrm{P}<0.01)$. In conclusion, visnagin may play a protective role in ischemia/reperfusion injury by inducing autophagy and reducing apoptosis.
\end{abstract}

Key words: Visnagin; ischemia/reperfusion (IR); autophagy; apoptosis.

Correspondence: Hai-rong Fu, Department of Basic Medicine, Three Gorges Medical College, Chongqing, 404120, China. E-mail: lemonfhr@126.com

Contributions: HF, conceived and designed research; HF, LP, YZ, performed experiments; HF, XL, BF, YZ, analyzed data; HF, YZ, BF drafted manuscript; HF, XL, YZ, LP, revised manuscript. All authors read and approved final version of manuscript.

Conflict of interest: The authors declare no conflict of interest.

Availability of data and materials: The data used to support the findings of this study are available from the corresponding author upon request.

Ethical approval: The Animal Research Ethics Committee of the Three Gorges Medical College approved the animal use protocol listed below. 


\section{Introduction}

Acute myocardial infarction (AMI) is the leading cause of coronary heart disease. Currently, the goal for healthcare professionals in management of AMI is to help early reperfusion in the ischemic area, such as percutaneous coronary intervention or thrombolytic therapy. Although these treatments can prevent a certain degree of myocardial damage, ischemia/reperfusion (IR) damage may still occur. This leads to cardiac function loss and myocardial cell death. ${ }^{1}$ Therefore, to limit the extent of IR damage can potentially improve the treatment of AMI.

Visnagin is furanochromone and one of the main compounds of Ammi visnaga L. (syn. khella) that had been widely used to treat nephrolithiasis in Ancient Egypt. ${ }^{2}$ It was found that intravenous injection of visnagin lowered the blood pressure with no change in the heart rate. ${ }^{3}$ It was also found that visnagin and its related compounds such as khellin and vesnadin can be used to treat angina pectoris since they play an important role in relaxing peripheral blood vessels and coronary arteries. ${ }^{4}$ On the other hand, it was found that visnadin effectively normalized the electrocardiogram of ischemic myocardia in a dog. ${ }^{5}$ Visnagin has been shown to prevent calcium ions from flowing into cells by inhibiting $\mathrm{Ca}^{2+}$ channels, thereby inhibiting vasoconstriction and lowering blood pressure. ${ }^{6}$ Recent studies have found that visnagin can reduce the apoptosis of ovarian follicular tissue in rats. ${ }^{7}$ In addition, visnagin has also been reported that it can be used to treat urolithiasis and hypertriglyceridemia. ${ }^{6}$ However, the protective effect of visnagin on myocardial ischemia/reperfusion injury has not been confirmed.

Autophagy is a one of the protective mechanisms of cells to multiple stressors. The rough endoplasmic reticulum (ER) lacking associated ribosomes engulfs dysfunctional proteins and organelles to form autophagosomes. Autophagosomes fuse with the lysosome to release lysosomal enzymes, thereby degrading engulfed autophagosome contents, and finally release metabolites. $^{8}$

In this study, we found that intravenous injection of visnagin improved cardiac function and reduced infarct size, after myocardial ischemia/reperfusion injury in rats. In addition, the apoptosis was inhibited since the expression of microtubule-associated protein 1A/1B-light chain 3 (LC3)-II and the number of autophagosomes increased. Therefore, we hypothesized that visnagin may be a potential new treatment for ischemia-reperfusion injury.

\section{Materials and Methods}

\section{Animals and reagents}

The male SD rats (250-300 g, SPF) were provided by the Experimental Animal Center of Chongqing Medical University (Certificate number: SCXK (Yu) 2017-0005).

The following reagents were used: visnagin, horseradish peroxidase (HRP)-labeled goat anti-mouse IgG, Ponceau S solution (Sigma-Aldrich, St. Louis, MO, USA); chloral hydrate, 4\% paraformaldehyde (Shan Pu Chemical Co., Ltd., Shanghai, China), TUNEL kit (Toyobo Co., Osaka, Japan); DAB kit (Zhongshan Goldenbridge Biotechnology, Co., Ltd., Beijing, China); LC3 antibody (Abcam, Cambridge, UK). Reagent grade is generally equal to analytical grade.

\section{Rat model}

SD rats were randomly divided into 3 groups including sham, ischemia / reperfusion group (IR), ischemia/reperfusion with vis- nagin $(n=10)$. The rats in the sham operation group were only threaded and not ligated. The left anterior descending coronary arteries were ligated in the rats in the IR group. After $45 \mathrm{~min}$ of ischemia, reperfusion was performed for $24 \mathrm{~h}$. After the IR model was established for $30 \mathrm{~min}$, rats received saline $(3 \mathrm{~mL} / \mathrm{kg}$ ) (sham group) and saline ( $3 \mathrm{~mL} / \mathrm{kg}$ ) (IR group), visnagin $(2 \mathrm{mg} / \mathrm{kg})$ (IR + visnagin group) (dissolved in saline), all of which were injected via the tail vein. All rats were sacrificed after the operation.

\section{Analysis of cardiac function}

Cardiac function was measured by hemodynamics and echocardiography. After the IR model was established, rats were injected with saline (sham and IR groups) and visnagin (IR + visnagin group) through the tail vein in each group. Two weeks after the injection, the rats were anesthetized, and then echocardiography was performed through the thorax with a $30-\mathrm{MHz}$ probe. Two weeks after the intravenous injection of the drug in all rats, the catheter was inserted into the left ventricle via the right common carotid artery. The pressure transducer is connected to the MS2000 biosignal sensor to record cardiac function data such as left ventricular systolic pressure (LVSP), left ventricular end diastolic pressure (LVEDP), and the rate of change in pressure with time $(\mathrm{dp} / \mathrm{dt})$ of left ventricular.

\section{Myocardial infarction diagnosis}

The anterior descending branch was ligated again after $24 \mathrm{~h}$ reperfusion, $2 \mathrm{~mL}$ of $2 \%$ Evans blue (Sigma-Aldrich) was injected from the femoral vein. The heart was then immediately removed, sliced into serial transverse sections, and placed in $1 \%$ of $2,3,5-$ triphenyltetrazolium chloride (TTC) for $15 \mathrm{~min}$ at $37^{\circ} \mathrm{C}$. Then, it was fixed in $10 \%$ methanol solution for 24 to $48 \mathrm{~h}$. Therefore, the non-ischemic myocardial areas were stained blue, non-ischemic areas and areas at risk (AAR) was stained red, and infarcted was stained white. Each slice was scanned and analyzed by Image-Pro Plus 6.0 image analysis software. Myocardial infarction area was calculated as a percentage $(\mathrm{AN} / \mathrm{AAR} \%)=($ infarct area $/$ area at risk) x $100 \%$.

\section{TUNEL assay and caspase activity}

Apoptosis was detected by TUNEL assay according to the instructions. All cardiomyocytes were labeled with DAPI, and apoptotic cells were labeled with green fluorescence. Apoptotic Index (AI) of cardiomyocytes $=$ number of apoptotic cells/total number of cardiomyocytes $x 10$. The activity of caspase- 3 and caspase- 9 was detected using caspase- 3 and caspase- 9 colorimetric activity assay kits. The caspase activities were detected with a spectrophotometer microplate reader at an absorbance of $405 \mathrm{~nm}$ in according to the instructions.

\section{Western blotting analysis}

Western blot was used to detect the LC3 protein expression in the myocardial tissue of left ventricular ischemic region. All proteins were extracted by radioimmunoprecipitation. We used the BCA protein detection kit to determine the protein concentration following the instructions. $50 \mu \mathrm{g}$ of protein was added and separated with $12 \%$ SDS-PAGE gel, then covered with PVDF membranes. Then, the membranes were blocked in 5\% skim milk powder in PBST $(0.05 \%$ Tween-20 in PBS) for $1 \mathrm{~h}$ at room temperature (RT). These membranes were incubated with primary antibody LC3 (1:1000) or GAPDH $(1: 1000)$ overnight at $4^{\circ} \mathrm{C}$. The membranes were washed with PBST for 10 min for 3 times, and then incubated with secondary antibody (rabbit IgG, $1: 500$ ) at $37^{\circ} \mathrm{C}$ for $1 \mathrm{~h}$. The membranes were washed with PBST for 10 min for 3 times. After that, the membranes were incubated with the ECL chemiluminescence solution for $3 \mathrm{~min}$, and exposed to the X-ray film in the dark room. We used 
quantity one software to analyze the protein expression. GAPDH was used as the internal reference. The relative protein expression level was accepted after normalization to the GAPDH.

\section{Transmission electron microscopy}

After injection of visnagin through the tail vein, the left ventricle was removed and fixed with $4 \%$ glutaraldehyde at RT for $24 \mathrm{~h}$, and then fixed with $2 \%$ osmic acid at RM for $2 \mathrm{~h}$. The tissue was dehydrated with a gradient of ethanol $(50,60,70,80,90,95$, and $100 \%$ ), and then transferred to a mixture of propylene oxide and embedding solution $(10 \mathrm{~mL})$ at RT for $1 \mathrm{~h}$, and then placed in an oven at $60^{\circ} \mathrm{C}$ to polymerize for $48 \mathrm{~h}$. The sections were cut as $80 \mathrm{~nm}$ thick with a manual Leica microtome and then doublestained with 5\% uranyl acetate and 5\% lead citrate at RT. Myocardial fibers and autophagosomes were observed under transmission electron microscopy (TEM) at 40-120kv.

\section{Statistical analysis}

All data are analyzed by Graphpad prism 8.0. The one-way ANOVA test was used for the comparison between two groups. $\mathrm{P}<0.05$ was considered statistically significant.

\section{Results}

\section{Visnagin can decrease cardiac dysfunction}

Echocardiography results showed that visnagin has a relief effect on cardiac dysfunction (Figure 1 A-C). Visnagin significantly reduced end-diastolic and end-systolic volume, increased left ventricular ejection fraction and shortened fraction $(\mathrm{P}<0.01)$ (Figure 1B). At the same time, visnagin can increase the left ventricular systolic pressure and the rate of change of left ventricular pressure $(\mathrm{dp} / \mathrm{dt})$ and reduce the left ventricular end-diastolic pressure $(\mathrm{P}<0.01)$ (Figure $1 \mathrm{C})$. Therefore, visnagin can improve systolic and diastolic function and stress, and play a role in relieving cardiac dysfunction. In addition, the AN/AAR $\%$ of the sham group and the IR group were significantly higher than those of the IR + visnagin group $(\mathrm{P}<0.05$ or $\mathrm{P}<0.01)$ (Figure $1 \mathrm{D})$. Moreover, Masson staining showed that visnagin could significantly inhibit fibrosis $(\mathrm{P}<0.01)$ (Figure 1E). The above results indicate that visnagin can relieve cardiac dysfunction, reduce the area of myocardial infarction, and prevent fibrosis.

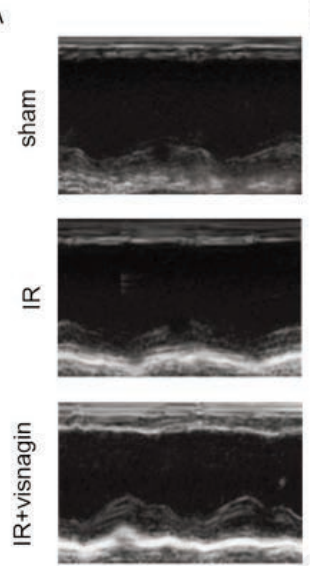

C

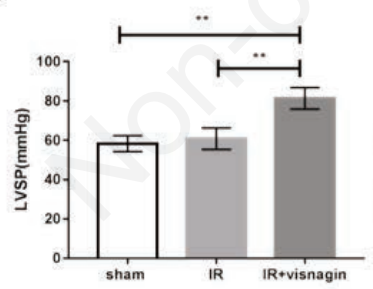

D

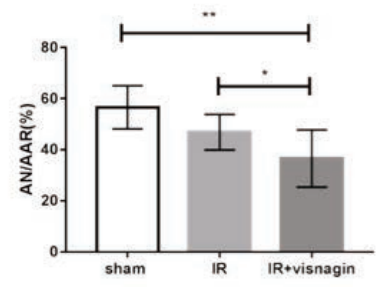

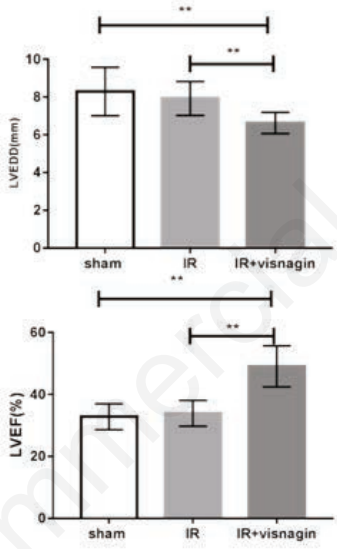
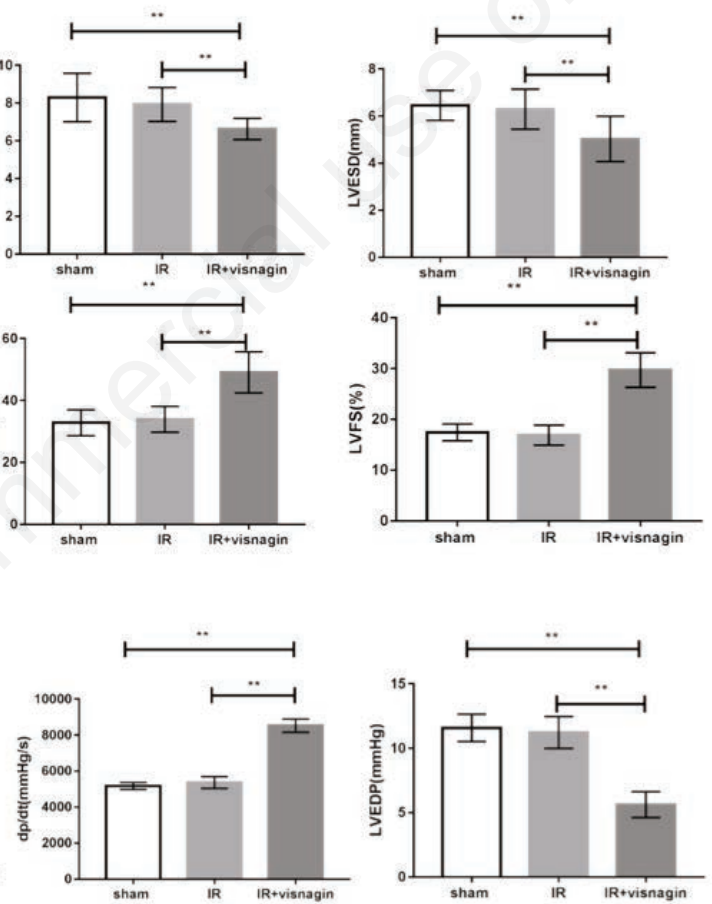

$E$

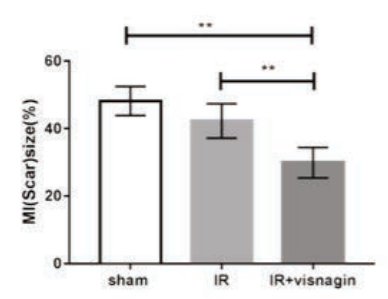

Figure 1. Visnagin ameliorated cardiac dysfunction 4 weeks after ischemia/reperfusion. A) Relief effect of visnagin on cardiac dysfunction shown by echocardiography. B) Effect of visnagin on LVEDD, LVESD, LVEFS. C) Systolic blood pressure was detected through the micromanometer catheter method, and the LVSP, dp/dt and LVEDP were calculated. D) Quantitative analysis of AN/AAR. E) Quantification of fibrosis. LVEDD, left ventricular end-diastolic dimension; LVESD, left ventricular end-systolic dimension; LVEF, left ventricular ejection fraction; LVFS, left ventricular fractional shortening; LVSP, left ventricular systolic pressure; LVEDP, left ventricular end-diastolic pressure; $\mathrm{dp} / \mathrm{dt}$, the rate of rise of left ventricular pressure; MI, myocardial infarction; AN, infarct area; AAR, area at risk; ${ }^{*} \mathbf{P}<0.05,{ }^{* *} \mathbf{P}<0.01$. 


\section{Visnagin can enhance autophagy in the ischemic area of cardiac ischemia/reperfusion injury}

Previous studies have shown that visnagin has a protective effect on myocardial cells in rats with ischemia/reperfusion injury, and the mechanism of the protective effect is further studied below. Autophagy is a self-protection mechanism produced when cells are under metabolic stress. LC3 is a marker of autophagy flow. Therefore, proteins extracted from the AAR were analyzed by western blotting. The results showed that the IR + visnagin group increased the LC3 protein expression (Figure $2 \mathrm{~A}, \mathrm{~B}$ ). In order to further prove that visnagin induced autophagy, immunofluorescence analysis was performed. The IR + visnagin group increased autophagy flow (Fig. 2C), which was consistent with the western blotting. TEM is the standard for detecting autophagic flow. After intervention with IR by visnagin, the ischemic area is analyzed by TEM. The number of double-layer structures in the IR + visnagin group was significantly improved (Figure 2D). The data indicated that visnagin can protect myocardial ischemia-reperfusion injury by inducing autophagy.

\section{Visnagin can inhibit myocardial cell apoptosis in ischemic area after myocardial IR injury}

Apoptosis is the most important mechanism of cell death in IR injury. Cell apoptosis was analyzed by TUNEL assay and detecting caspase activity. As shown in Figure 3 A,B, compared with the IR + visnagin group, the AI of the sham group and the IR group increased significantly. In addition, the caspase- 3 and caspase- 9 activities in the IR + visnagin group were significantly reduced, compared with the sham group and the IR group (Figure $3 \mathrm{C}, \mathrm{D}$ ). These results suggest that visnagin can protect cells by inhibiting apoptosis in myocardial ischemic injury.

\section{Discussion}

This study shows that visnagin has a protective effect on myocardial IR injury, which can reduce the area of myocardial infarction and improve cardiac dysfunction. In addition, the protective mechanism of visnagin on myocardium may be through induction of autophagy and inhibition of apoptosis.

Studies have shown that Shenfu injection reduced the infarct size and pathological changes of myocardial ischemia/reperfusion in rats, and also reduced the LC3II/LC3I ratio and Beclin1 protein expression level in myocardial tissue. ${ }^{9}$ The present study showed that intravenous injection of visnagin during reperfusion can significantly reduce the size of myocardial infarction area after $24 \mathrm{~h}$ reperfusion. Meanwhile, it can reduce cardiac dysfunction and
A

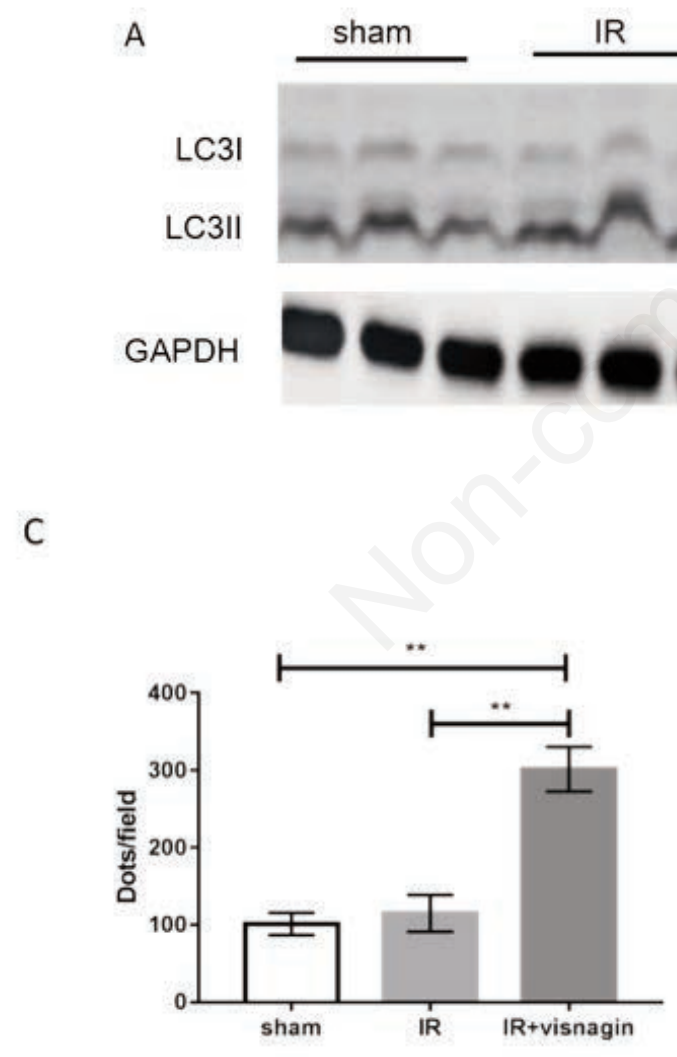

B

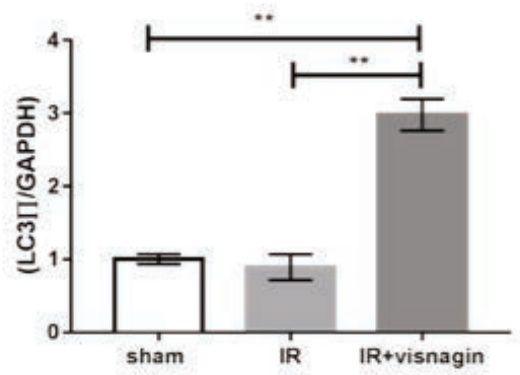

D

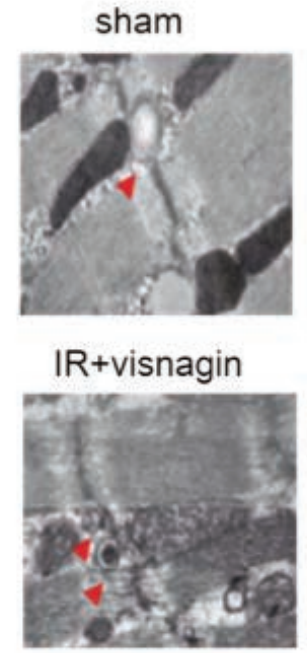

IR

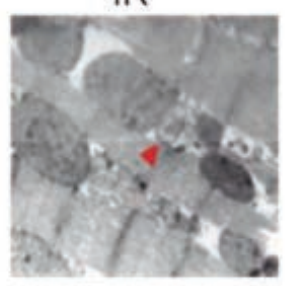

Figure 2. Visnagin enhanced the rate of autophagy in the ischemic region after ischemia/reperfusion. A) Western blotting was performed for the detection of LC3. B) Quantification of the expression of LC3II relative to GAPDH. C) Positive dots of LC3 (D)detection of double membrane autophagosomes with transmission electron microscopy. ${ }^{*} \mathbf{P}<0.05,{ }^{* *} \mathbf{P}<\mathbf{0 . 0 1}$. 
inhibit myocardial fibrosis after 4 weeks reperfusion. These conclusions suggest that visnagin is a potential treatment for myocardial IR injury.

Visnagin is a natural compound extracted from the fruit of Ammi visnaga. ${ }^{10}$ It has been reported that visnagin relaxes blood vessels by inhibiting the contraction of vascular smooth muscle in multiple sites and inhibiting the hydrolysis activity of cyclic nucleotide phosphodiesterase (PDE) isoenzymes. ${ }^{11}$ In addition, it has also been reported that visnagin can inhibit the nitric oxide in microglia induced by lipopolysaccharide (LPS), and also reduce the proinflammatory cytokines such as IL-1, IL-6 and TNF- $\alpha .{ }^{12}$ Moreover, visnagin can improve the cardiotoxicity induced by adriamycin by inducing malate dehydrogenase in mitochondria to trigger tricarboxylic acid cycle and promote myocardial metabolism. ${ }^{13,14}$ In an anti-tumor studies, Aydogmus-Ozturk and other researchers have found that visnagin can inhibit the proliferation of human malignant melanoma cells by inducing intracellular ROS production and activating pro-apoptotic pathways. ${ }^{15,16}$ Vrzal et al. demonstrated that visnagin can inhibit the growth, differentiation and migration of tumor cells in human hepatocellular carcinoma by promoting aromatic hydrocarbon receptor (AHR) signaling. ${ }^{17}$

Autophagy is a self-protecting cellular program in which cells respond to stress. LC3 is an important factor regulating cell autophagy and a specific marker for monitoring autophagy, and its expression level is closely related to autophagy activity. ${ }^{18}$ In this study, we found that the IR + visnagin group increased the LC3-II expression level. Studies have shown that autophagy can alleviate metabolic crisis in myocardial IR injury through ATP production. ${ }^{19}$ Low levels of ATP can induce myocardial autophagy by activating the adenosine monophosphate-activated protein kinase (AMPK) / mammalian target of rapamycin (mTOR) -Uncoordinated-51-like kinase 1 (ULK1) pathway. ${ }^{19}$ The recovery of energy by cardiac autophagy is essential for the survival of cardiomyocytes. In addition, autophagy promotes protein homeostasis in IR injury. ${ }^{20}$ Once autophagy is activated during IR damage, it can remove toxic ubiquitinated proteins from cells and reduce protein aggregation. ${ }^{21}$ Increased autophagy activity can compensate the ubiquitin-proteasome system and promote proteolytic stability. ${ }^{22}$ In addition, in the case of excessive production of reactive oxygen species and inflammatory response during IR injury, the induction of autophagy may remove dysfunctional mitochondria and recycle waste to maintain energy balance, prevent mitochondria damage from releasing cytotoxic substances, and promote myocardial cells survival. ${ }^{23,24}$ The cardioprotective effect of autophagy was also demonstrated in a way to prevent ischemia/reperfusion damage. ${ }^{25}$

In contrast to autophagy, it has been shown that apoptosis may be related to the pathogenesis of myocardium in IR injury and lead to myocardial cell apoptosis. ${ }^{26}$ In this study, the AI of the IR +

A
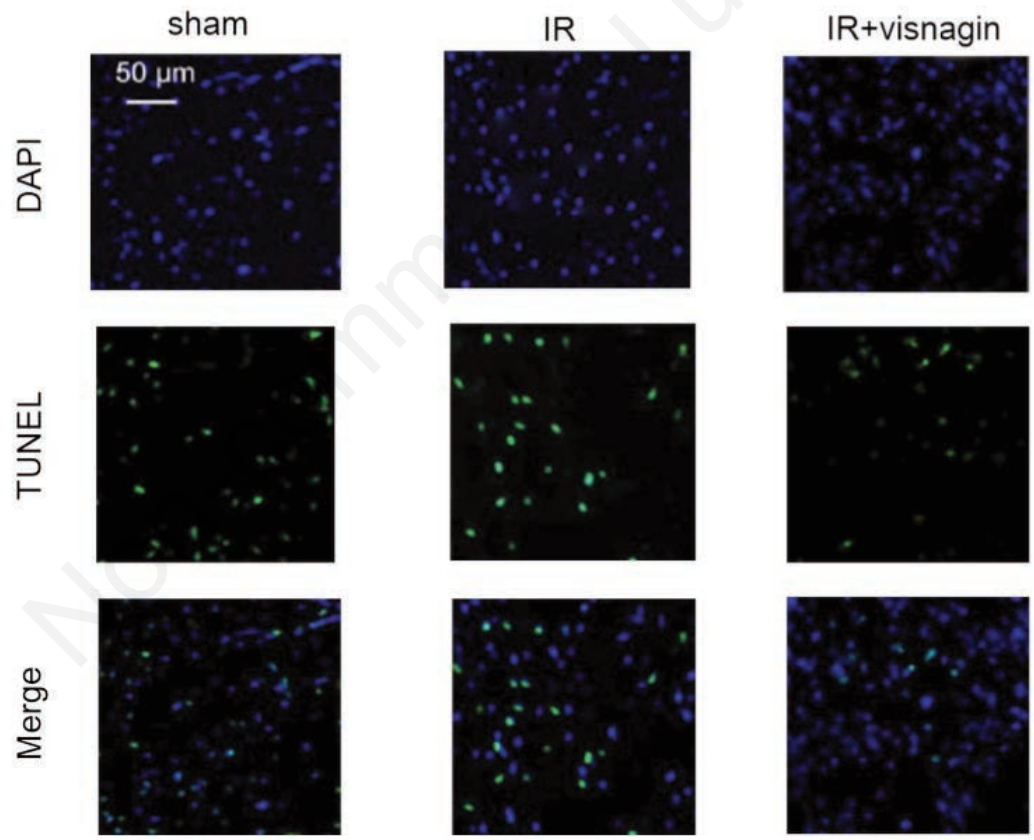

B

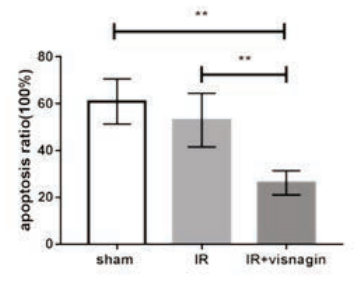

C

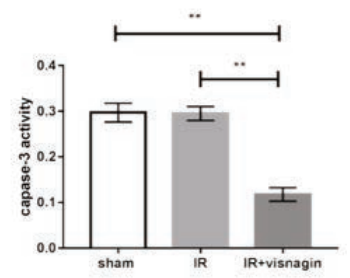

D

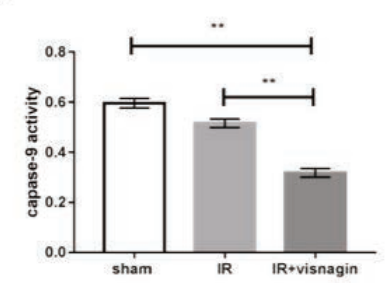

Figure 3. Visnagin inhibited apoptosis in the ischemic region subsequent to IR. A) Cell apoptosis detected by TUNEL assay. B) Quantification of apoptotic cells. C) Caspase-3 activity of the myocardium subsequent to IR. D) Caspase-9 activity of the myocardium subsequent to IR. IR, ischemia/reperfusion; ${ }^{* *} \mathrm{P}<0.01$. 
Visnagin group was significantly increased, and caspase-3 and caspase-9 activities were significantly reduced. Studies have demonstrated that apoptotic-like morphological changes are observed during IR injury, suggesting that cardiomyocyte apoptosis is initiated. ${ }^{27}$ Oxygen free radicals, mitochondrial damage, and calcium accumulation are potential inducers of cardiomyocyte apoptosis in IR injury. The generation and accumulation of oxygen free radicals finally activates the chain reaction leading to nucleic acid destruction. ${ }^{28}$ Mitochondrial damage may help mitochondria to release apoptosis-inducing factors, including apoptotic protease activating factor 1, which can promote caspase-induced apoptosis. The accumulation of $\mathrm{Ca}^{2+}$ in mitochondria may activate the mitochondrial permeability transition pores and help release cytochrome $\mathrm{C}$ into the cytoplasm, thereby activating caspase to induce apoptosis. ${ }^{29}$

It has shown that AHR signaling is involved in the regulation of autophagy and apoptosis. AHR is one of upstream factors of the Beclin1/Bcl2 complex; it can inhibit the interaction between Beclin1 and $\mathrm{Bcl} 2$, thereby promoting the release of Beclin1. However, AHR may also promote the development of the autophagy-related gene 14 (Atg14) -Vps34 complex to the Atg14Vps34-ULK1 complex, which can act as an activator of autophagy. ${ }^{30,31}$ AHR is also an activator of anti-apoptotic proteins (Bcl2, Bcl-XL and survivin) and an inhibitor of pro-apoptotic proteins (BAX, Bcl2 and P53). ${ }^{32}$ Visnagin has also been reported to be an inducer of AHR. ${ }^{33}$ In this study, we found visnagin can promote autophagy and inhibit apoptosis in ischemic areas. It suggests that the protective mechanism of visnagin may be through inducing autophagy and inhibiting apoptosis.

Collectively, visnagin has been proven to provide cardioprotection. Visnagin can reduce the size of myocardial infarction and improve cardiac dysfunction by inducing autophagy and inhibiting apoptosis. Studies on the cardioprotective effects of visnagin have shown that visnagin may be a new potential treatment for IR injuries, but since there is a balance point of cardiovascular protection between apoptosis and autophagy, the balance point of the protective effect of visnagin on myocardial ischemia-reperfusion injury should be further explored, to provide a basis for development and utilization of drugs for ischemic heart disease.

\section{Acknowledgments}

The authors express their gratitude to Acesci Biomed in Wuhan for the proof-reading and grammatical errors correction. This work belongs to Academic Program of Traditional Medicine supported by Chongqing municipal health and Health Committee (ZY201703078)

\section{References}

1. Liu XM, Yang ZM, Liu XK. Fas/FasL induces myocardial cell apoptosis in myocardial ischemia-reperfusion rat model. Eur Rev Med Pharmacol Sci 2017;21:2913-8.

2. Asnani A, Zheng B, Liu Y, Wang Y, Chen HH, Vohra A, et al. Highly potent visnagin derivatives inhibit cyp1 and prevent doxorubicin cardiotoxicity. JCI Insight 2018;3:e96753.

3. Duarte J, Perez-Vizcaino F, Torres AI, Zarzuelo A, Jimenez J. Vasodilator effects of visnagin in isolated rat vascular smooth muscle. Eur J Pharm 1995,286,115-22.

4. Duarte J, Lugnier C, Torres AI, Perez-Vizcaino F, Zarzuelo A, Tamargo J. Effects of visnagin on cyclic nucleotide phosphodiesterases and their role in its inhibitory effects on vascular smooth muscle contraction. Gen Pharmacol 1999;32:71-4.

5. Duarte J, Torres AI, Zarzuelo AI. Cardiovascular effects of visnagin on rats. Planta Med 2000;66:35-9.

6. Bhagavathula AS, Mahmoud Al-Khatib AJ, Elnour AA, Al Kalbani NM, Shehab A. Ammi Visnaga in treatment of urolithiasis and hypertriglyceridemia. Pharmacognosy Res 2014;7:397-400

7. Abu-Hashem AA, El-Shazly M. Synthesis, reactions and biological activities of furochromones: a review. Eur J Med Chem 2015;90:633-65.

8. Pasari LP, Khurana A, Anchi P, Aslam Saifi M, Annaldas S, Godugu C. Visnagin attenuates acute pancreatitis via Nrf2/NFkB pathway and abrogates associated multiple organ dysfunction. Biomed Pharmacother 2019;112:108629.

9. Wu D, Zhang K, Hu P. The role of autophagy in acute myocardial infarction. Front Pharmacol 2019;10:551.

10. Jia HL,LU CQ, Wang J, Ren DD, Chen YQ.Effect of shenfu injection on alleviating myocardial ischemia-reperfusion injury in rats by regulating autophagy. Chin J Immunol 2019;35:2213-20.

11. Khalil HS, Sedky NK, Amin KM, Abd Elhafez OM, Arafa RK. Visnagin and benzofuran scaffold-based molecules as selective cyclooxygenase-2 inhibitors with anti-inflammatory and analgesic properties: design, synthesis and molecular docking. Future Med Chem 2019;11:659-76.

12. Asnani A, Zheng B, Liu Y, Wang Y, Chen HH, Vohra A, et al. Highly potent visnagin derivatives inhibit Cyp1 and prevent doxorubicin cardiotoxicity. JCI Insight 2018;3.

13. Lee JK, Jung JS, Park SH, Park SH, Sim YB, Kim SM, et al. Anti-inflammatory effect of visnagin in lipopolysaccharidestimulated BV-2 microglial cells. Arch Pharm Res 2010;33:1843-50.

14. Haug KG, Weber B, Hochhaus G, Butterweck V. Nonlinear pharmacokinetics of visnagin in rats after intravenous bolus administration. Eur J Pharm Sci 2012;45:79-89.

15. Jahan H, Choudhary MI, Shah Z, Khan KM, Rahman AU. Derivatives of 6-nitrobenzimidazole inhibit fructose-mediated protein glycation and intracellular reactive oxygen species production. Med Chem 2017;13:577-84.

16. Aydogmus-Ozturk F, Jahan H, Beyazit N, Gunaydin K, Choudhary MI. The anticancer activity of visnagin, isolated from Ammi visnaga L., against the human malignant melanoma cell lines, HT 144. Mol Biol Rep 2019;46:1709-14.

17. Vrzal R, Frauenstein K, Proksch P, Abel J, Dvorak Z, Haarmann-Stemmann T. Khellin and visnagin differentially modulate AHR signaling and downstream CYP1A activity in human liver cells. PLoS One 2013;8:e74917.

18. Kuma A, Matsui M, Mizushima N. LC3, an autophagosome marker, can be incorporated into protein aggregates independent of autophagy: caution in the interpretation of LC3 localization. Autophagy 2007;3:323-8.

19. Gatica D, Chiong M, Lavandero S, Klionsky DJ. Molecular mechanisms of autophagy in the cardiovascular system. Circ Res 2015;116:456-67.

20. Liu Y, Asnani A, Zou L, Bentley VL, Yu M, Wang Y, et al. Visnagin protects against doxorubicin-induced cardiomyopathy through modulation of mitochondrial malate dehydrogenase. Sci Transl Med 2014;6:266ra170

21. Zuo Z, Zuo PF, Sheng ZL, Wang X, Ding JD, Ma GS. Tetramethylprazine attenuates myocardial ischemia/reperfusion injury through modulation of autophagy. Life Sci 2019;239:117016.

22. Hu C, Tian Y, Xu H, Pan B, Terpstra EM, Wu P, et al. Inadequate ubiquitination-proteasome coupling contributes to myocardial ischemia-reperfusion injury. $\mathrm{J}$ Clin Invest 
2018;128:5294-306.

23. Parry TL, Willis MS. Cardiac ubiquitin ligases: Their role in cardiac metabolism, autophagy, cardioprotection and therapeutic potential. Biochim Biophys Acta 2016;1862:2259-69.

24. Adams B, Mapanga RF, Essop MF. Partial inhibition of the ubiquitin-proteasome system ameliorates cardiac dysfunction following ischemia-reperfusion in the presence of high glucose. Cardiovasc Diabetol 2015;14:94.

25. Tan Z, Liu H, Song X, Ling Y, He S, Yan Y, et al. Honokiol post-treatment ameliorates myocardial ischemia/reperfusion injury by enhancing autophagic flux and reducing intracellular ROS production. Chem Biol Interact 2019;307:82-90.

26. Kooti W, Servatyri K, Behzadifar M, Asadi-Samani M. Effective medicinal plant in cancer treatment, part 2: review study. J Evid Based Complementary Altern Med 2017;22:982-95.

27. Al-Salam S, Hashmi S. Myocardial ischemia reperfusion injury: Apoptotic, inflammatory and oxidative stress role of galectin-3. Cell Physiol Biochem 2018;50:1123-39.

28. Cai Y, Ying F, Liu H, Ge L, Song E, Wang L, et al. Deletion of Rap1 protects against myocardial ischemia/reperfusion injury through suppressing cell apoptosis via activation of STAT3 signaling. FASEB J 2020;34:4482-96.
29. Liu L, Wang Q, Zhao B, Wu Q, Wang P. Exogenous nicotinamide adenine dinucleotide administration alleviates ischemia/reperfusion-induced oxidative injury in isolated rat hearts via Sirt5-SDH-succinate pathway. Eur J Pharmacol 2019;858:172520.

30. Zhang H, Wang X, Ma Y, Shi Y. The effect of ginsenoside RB1, diazoxide, and 5-hydroxydecanoate on hypoxia-reoxygenation injury of $\mathrm{H} 9 \mathrm{C} 2$ cardiomyocytes. J Evid Based Complement Alternat Med 2019;2019:6046405.

31. Han J, Park JC, Hagiwara A, Park HG, Lee JS. Identification of the full 26 cytochrome P450 (CYP) genes and analysis of their expression in response to benzo[alpha]pyrene in the marine rotifer Brachionus rotundiformis. Comp Biochem Physiol Part D Genomics Proteomics 2019;29:185-92.

32. Miao Y, Lv Q, Qiao S, Yang L, Tao Y, Yan W, et al. Alpinetin improves intestinal barrier homeostasis via regulating AhR/suv39h1/TSC2/mTORC1/autophagy pathway. Toxicol Appl Pharmacol 2019;384:114772.

33. Das DN, Naik PP, Mukhopadhyay S, Panda PK, Sinha N, Meher BR, et al. Elimination of dysfunctional mitochondria through mitophagy suppresses benzo[a]pyrene-induced apoptosis. Free Radic Biol Med 2017;112:452-63.

Received for publication: 23 March 2020. Accepted for publication: 30 May 2020.

This work is licensed under a Creative Commons Attribution-NonCommercial 4.0 International License (CC BY-NC 4.0).

(C) Copyright: the Author(s), 2020

Licensee PAGEPress, Italy

European Journal of Histochemistry 2020; 64(s2):3131

doi:10.4081/ejh.2020.3131 